\title{
IV-VI SEMIMAGNETIC SEMICONDUCTORS WITH RARE EARTH IONS*
}

\author{
T. STORY \\ Institute of Physics, Polish Academy of Sciences \\ Al. Lotników 32/46, 02-668 Warsaw, Poland
}

The electronic and magnetic properties as well as the applications of IV-VI semiconductors with rare earth ions of $\mathrm{Eu}$ and $\mathrm{Yb}$ are briefly discussed. The detailed analysis is presented of the recent experimental investigations of the magnetic and transport properties of IV-VI semimagnetic crystals with $\mathrm{Gd}$. It concerns, in particular, the effect of Fermi level position controlled $f-f$ exchange interaction between $\mathrm{Gd}$ ions in $\mathrm{Sn}_{1-x} \mathrm{Gd}_{x} \mathrm{Te}$.

PACS numbers: 75.20.Ck, 75.30.Et

\section{Introduction}

The solubility limit of rare earth ions is limited to doping level in $\mathrm{Si}$ as well as in III-V and in II-VI compound semiconductors. IV-VI semiconductors offer the unique possibility to study the mixed semiconducting bulk crystals and MBE-grown thin layers and structures with the concentration of, e.g., Eu, Gd or $\mathrm{Yb}$ typically up to 10 at.\% [1].

The IV-VI crystals with Eu are known to be effective mid-infrared tunable detectors and lasers. The laser with $\mathrm{PbTe}-\mathrm{PbEuSeTe}$ heterostructure currently holds the record for the working temperature of the continuous wave operation mid-infrared semiconductor laser $(T=223 \mathrm{~K}$ [2]). The layer of IV-VI semiconductor with Eu may serve both as the active layer of the device or as the layer providing electrical and optical confinement $[2,3]$. The physical property relevant for the applications of these crystals is the rapid increase in the energy gap with the increasing Eu content $\left(\mathrm{d} E_{\mathrm{g}} / \mathrm{d} x=(30 \div 50) \mathrm{meV} /\right.$ at.\% [4-6]). Both $\mathrm{Pb}_{1-x} \mathrm{Eu}_{x} \mathrm{Te}$ and $\mathrm{Pb}_{1-x} \mathrm{Eu}_{x} \mathrm{Se}$ are direct gap semiconductors with good luminescent properties.

IV-VI semiconductors such as $\mathrm{PbTe}, \mathrm{PbSe}$ or SnTe are strongly degenerated materials with a narrow energy gap [7]. The characteristic property of these crystals is a very high concentration of vacancies. Metal vacancies are known to be acceptor centers whereas non-metal vacancies show the donor character. As the energy levels of these defects are located in the conduction band (above the Fermi level), there is

*This work was supported in part by the Committee for Scientific Research grant 2 P03 B 10308. 
no activation energy for the generation of the conducting carriers. It leads to high, temperature independent, conductivity with the conducting carrier concentrations of the order of $10^{20}-10^{21} \mathrm{~cm}^{-3}$ for SnTe and $10^{17}-10^{19} \mathrm{~cm}^{-3}$ for PbTe. The intermediate carrier concentrations can be obtained in mixed $\mathrm{Pb}_{1-y} \mathrm{Sn}_{y} \mathrm{Te}$ alloys. There exists a possibility to control the number of vacancies (i.e., the concentration of carriers) by an isothermal annealing. In SnTe, for the hole concentration $p \geq 2 \times 10^{20} \mathrm{~cm}^{-3}$, two valence bands are populated: the band of light holes located at the $L$-point of the Brillouin zone and the band of heavy holes located at the $\Sigma$-point of the Brillouin zone and shifted down from the top of the $L$-band by about $0.15-0.2 \mathrm{eV}[7]$.

IV-VI semiconductors with rare earth ions belong to the family of semimagnetic (diluted magnetic) semiconductors [1,4]. These are the mixed crystals of semiconducting II-VI or IV-VI compounds and magnetic semiconductors such as, e.g., MnTe, GdTe or EuSe. The magnetic ions substitute $\mathrm{Sn}^{2+}$ or $\mathrm{Pb}^{2+}$ ions in the rock salt lattice of IV-VI semiconductors. The investigations of the magnetic properties of Mn-based IV-VI semimagnetic semiconductors revealed the essential role of conducting carriers. The $\mathrm{Pb}_{1-x-y} \mathrm{Sn}_{y} \mathrm{Mn}_{x} \mathrm{Te}$ and $\mathrm{Sn}_{1-x} \mathrm{Mn}_{x} \mathrm{Te}$ crystals with carrier concentration $p>p_{\mathrm{t}}=3 \times 10^{20} \mathrm{~cm}^{-3}$ are ferromagnets or spin glasses at liquid helium temperatures [8-12]. The crystals with $p<p_{\mathrm{t}}$ show only very weak antiferromagnetic interactions between $\mathrm{Mn}$ ions. The ferromagnetic Curie temperature of $\mathrm{PbSnMnTe}$ and SnMnTe depends on the carrier concentration in a threshold-like way [8]. This dependence is explained within the frames of the Ruderman-Kittel-Kasuya-Yoshida (RKKY) indirect exchange interaction taking into account the contribution from both the light holes and the heavy holes $[8,11]$.

The complete analysis of the physical properties of IV-VI semimagnetic semiconductors with rare earth ions is beyond the'scope of this paper. We will focus on the experimental studies indicating the mutual influence of the electronic and the magnetic properties of these crystals. We will briefly discuss the results of the recent activities in IV-VI semicondurtors with $\mathrm{Eu}$ and with $\mathrm{Yb}$. The more detailed analysis will be given for the results of the experimental investigations of the magnetic and the electronic properties of $\mathrm{Sn}_{1-x} \mathrm{Gd}_{x} \mathrm{Te}$ crystals that reveal the effect of the strong resonant-like carrier concentration dependence of both the strength of the antiferromagnetic $f-f$ exchange interactions between $\mathrm{Gd}$ ions, and the mobility of conducting holes [13-16].

\section{IV-VI semiconductors with Eu and with Yb}

Owing to the applications, most of the experimental work on $\mathrm{Pb}_{1-x} \mathrm{Eu}_{x} \mathrm{Se}$, $\mathrm{Pb}_{1-x} \mathrm{Eu}_{x} \mathrm{Te}$ and their alloys was devoted to the investigations of optical and magneto-optical properties [4-6]. It includes the study of the optical nonlinear effect of coherent anti-Stokes Raman scattering (CARS) being the source of very detailed information on the band structure parameters of IV-VI semiconductors [4]. The good quantitative description of the experimental results was obtained within the frames of Mitchell-Wallis model supplemented with the $s p-f$ (conducting carrier - magnetic moment) exchange interaction terms well known in semimagnetic semiconductors. This model is discussed in Ref. [4] by Bauer, Pascher and Zawadzki. 
The magneto-optical experiments have also clearly demonstrated the role of the $s p-f$ exchange interactions and allowed for the determination of the $s p-f$ exchange integrals [4]. The general conclusion is that due to the very strong localization of the $4 f$ electrons the $s-f(p-f)$ exchange coupling is rather weak with the exchange integrals of the order of $10-20 \mathrm{meV}$ [4]. The small magnitude of the $s p-f$ exchange integrals in IV-VI semiconductors is also confirmed by the results of the early electron paramagnetic resonance (EPR) work by Sperlich and Urban [17]. The current understanding of the physical origin of this coupling is discussed by Dietl et al. [18].

The studies of the magnetic properties initiated by Górska et al. revealed only rather weak antiferromagnetic $f-f$ exchange interactions between Eu ions in $\mathrm{PbEuTe}, \mathrm{PbEuSe}$ and $\mathrm{SnEuTe}$ [19]. The detailed analysis of the $f-f$ exchange interactions between Eu ions in PbEuSe was recently done in Ref. [20] where the $I_{f-f}$ exchange integrals were determined from the analysis of the so-called magnetization steps. The $f-f$ exchange integral in $\mathrm{Pb}_{1-x} \mathrm{Eu}_{x} \mathrm{Te}$ was recently shown to exhibit an intriguing composition dependence. The effect is attributed to the composition induced shift of the top of the valence band of $\mathrm{Pb}_{1-x} \mathrm{Eu}_{x} \mathrm{Te}$ with respect to the energy level of $\mathrm{Eu} 4 f$ electrons responsible for the magnetic properties of these crystals [21].

The progress was also achieved in the field of EPR studies of Eu ions in IV-VI semiconductors. The standard (for $\mathrm{Eu}^{2+}$ ) 7-line anisotropic spectrum was experimentally observed and quantitatively analyzed both in bulk crystals [22] as well as in thin layers grown by molecular beam epitaxy [23].

The important physical problem which influences the understanding of both the magnetic and the electronic properties of IV-VI semiconductors with Eu is the location of the density of states related to the $4 f$ electronic states of Eu within the frames of the band structure of these semiconductors. The recent measurements of the magnetic properties [19-21], electron paramagnetic resonance [22, 23], photoemission [24] and transport [25] properties established the position of these states below the top of the valence band. One has to notice that these states are still relatively close to the top of the valence band and their composition induced shift may influence the electronic properties of $\mathrm{Pb}_{1-x} \mathrm{Eu}_{x} \mathrm{Te}[21,25]$.

The other rare earth ion which has recently raised a lot of activity is $\mathrm{Yb}$. The compositions up to 20 at.\% are reported in $\mathrm{Pb}_{1-x} \mathrm{Yb}_{x} \mathrm{Te}$ bulk crystals grown by Bridgman method as well as in epitaxial layers [26-28]. In its $\mathrm{Yb}^{2+}$ charge state $\mathrm{Yb}$ forms a system with $144 f$ electrons. The $4 f$ electron shell is then completely filled giving no contribution to the magnetic moment of the ion and, of course, showing no electron paramagnetic resonance. In IV-VI semiconductors $\mathrm{Yb}$ is usually in this $(2+)$ charge state being electrically neutral and showing only diamagnetic response to the magnetic field. Recent experimental studies of the electron paramagnetic resonance and magnetization in $\mathrm{Pb}_{1-x} \mathrm{Yb}_{x} \mathrm{Te}, \mathrm{Pb}_{1-x} \mathrm{Yb}_{x} \mathrm{Se}$ [29] and $\mathrm{Pb}_{1-x-y} \mathrm{Ge}_{y} \mathrm{Yb}_{x} \mathrm{Te}[30]$ revealed also the presence of $\mathrm{Yb}^{3+}$ ions, easily recognizable due to their $S=1 / 2, L=3$ magnetic moment. These crystals show also very interesting electric properties. The composition induced metal-insulator transition is observed both in PbYbTe and in PbGeYbTe $[27,28,30]$. 
The problem of primary importance for the understanding of both magnetic and transport properties of the IV-VI crystals with $\mathrm{Yb}$ is the experimental determination of the position of the energy levels related to the $\mathrm{Yb} 4 f$ electrons and the identification of the microscopic origin of the defect centers responsible for the thermally activated transport observed in PbYbTe and in PbGeYbTe. The two ideas forwarded by now imply either the formation of the mixed valence $\mathrm{Yb}^{2+/ 3+}$ system with the $\mathrm{Yb}^{2+/ 3+}$ energy level located in the energy gap [30], or the composition induced shift of the energy states of native defects from the valence band into the energy gap [28].

\section{Magnetic and transport properties of SnGdTe}

The bulk crystals of IV-VI semiconductors with Gd are single phase crystalline materials up to about 9 at.\% of $\mathrm{Gd}$. In previous studies of $\mathrm{Sn}_{1-x} \mathrm{Gd}_{x} \mathrm{Te}$, $\mathrm{Pb}_{1-x} \mathrm{Gd}_{x} \mathrm{Te}$ and $\mathrm{Pb}_{1-x} \mathrm{Gd}_{x} \mathrm{Se}, \mathrm{Gd}$ was found to substitute $\mathrm{Sn}^{2+}$ or $\mathrm{Pb}^{2+}$ ions in the rock-salt lattice of IV-VI matrix as $\mathrm{Gd}^{3+}$ ion, being an electrically active (donor) center possessing the $S=7 / 2$ well-localized magnetic moment due to $4 f^{7}$ electrons. The magnetic properties of these crystals were found to be carrier concentration independent, in contrast to the magnetic properties of, related, $\mathrm{Sn}_{1-x} \mathrm{Mn}_{x} \mathrm{Te}$ crystals mentioned in the Introduction. The problem of the correlations between magnetic and electronic properties of SnGdTe was the subject of recent experimental work which will be reviewed below.

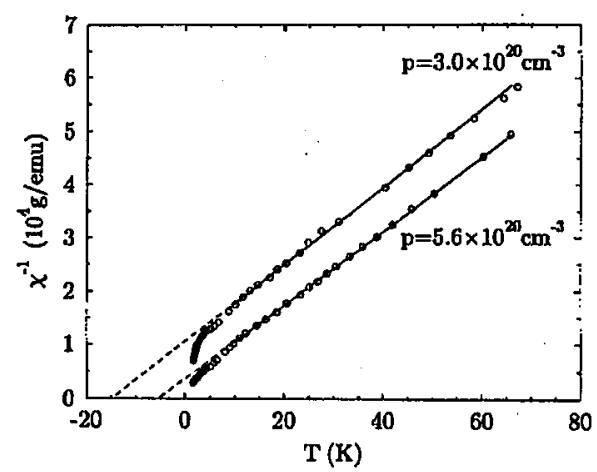

Fig. 1. The temperature dependence of the inverse of the magnetic susceptibility of $\mathrm{Sn}_{0.955} \mathrm{Gd}_{0.045}$ Te for two different carrier concentrations.

The typical temperature dependence of the inverse of the magnetic susceptibility of $\mathrm{Sn}_{1-x} \mathrm{Gd}_{x} \mathrm{Te}$ is presented in Fig. 1. The standard Curie-Weiss behavior is observed, as expected for the system of weakly interacting well-localized magnetic moments. The negative sign of the paramagnetic Curie temperature $(\Theta)$ indicates the presence of the antiferromagnetic $f-f$ interactions between $\mathrm{Gd}$ ions. The pair of samples presented in Fig. 1 is the same sample before and after annealing during which the concentration of carriers was reduced from $p=5.6 \times 10^{20} \mathrm{~cm}^{-3}$ to $p=3.0 \times 10^{20} \mathrm{~cm}^{-3}$ while the concentration of $\mathrm{Gd}$ remained unchanged, as indicated by the lack of the change of the Curie constant (determined by the slope 
of the $\chi^{-1}(T)$ plot). This experimental result illustrates the influence of the carrier concentration on magnetic properties of SnGdTe.

The carrier concentration dependence of $f-f$ exchange interactions between Gd ions in $\mathrm{Sn}_{1-x} \mathrm{Gd}_{x} \mathrm{Te}$ is presented in Fig. 2. The strength of this interaction is here characterized by the absolute value of the parameter $\theta / x$ which is related to the exchange integrals $I_{f f}(R)$ by the expression $k_{\mathrm{B}} \Theta / x=(2 / 3) S(S+1) \times$ $\sum z_{i} I_{f f}\left(R_{i}\right)$. The sum runs through all the magnetic neighbors $z_{i}$ located at distances $R_{i}$. One can notice that the difference in the value of the parameter $\Theta / x$ for different SnGdTe samples can be as large as an order of magnitude. The strong enhancement of the antiferromagnetic interspin coupling is observed for the samples with $0.025<x<0.05$ and with $p=(3 \pm 0.5) \times 10^{20} \mathrm{~cm}^{-3}$ only. The effect for the samples with $x>0.05$ or $x<0.025$ is much less pronounced. Also the samples with $0.025<x<0.05$ and with $p \gg 3 \times 10^{20} \mathrm{~cm}^{-3}$ show no enhancement of the $f-f$ exchange interaction.
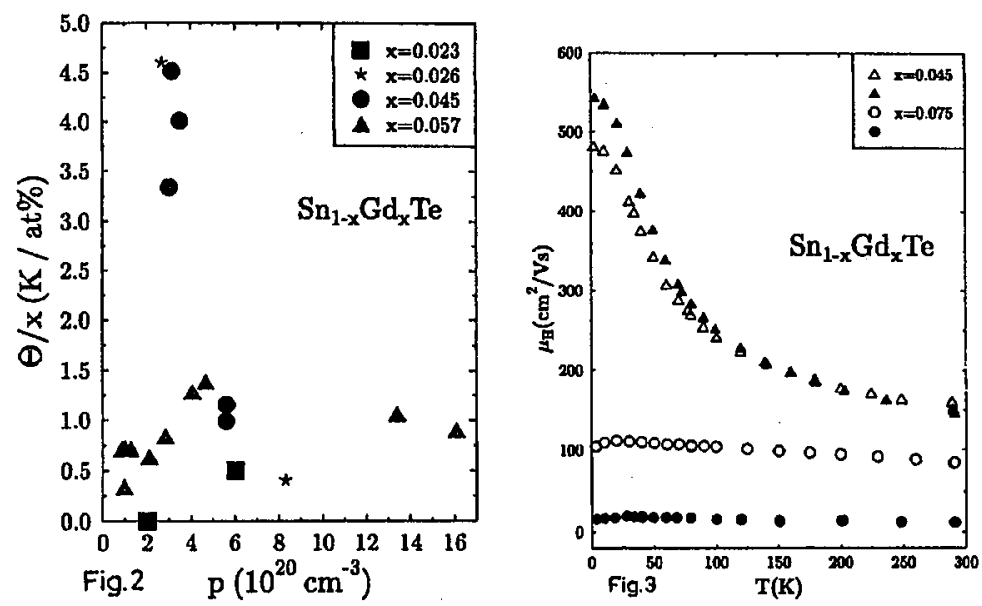

Fig. 2. The carrier concentration dependence of the normalized paramagnetic Curie temperature of $\mathrm{Sn}_{1-x} \mathrm{Gd}_{x} \mathrm{Te}$ with various $\mathrm{Gd}$ contents.

Fig. 3. The temperature dependence of the mobility of conducting holes (at $T=4 \mathrm{~K}$ ) in $\mathrm{Sn}_{1-x} \mathrm{Gd}_{x} \mathrm{Te}$ with various $\mathrm{Gd}$ compositions and carrier concentrations. Triangles show the data for the crystals with $x=0.045$ annealed in the atmosphere of Sn and exhibiting the enhanced magnetic interactions. Circles show the data for the annealed (open circles) and as-grown (full circles) crystals with $x=0.075$.

The measurements of transport properties of SnGdTe revealed that in the very same samples in which the large $\Theta / x$ is observed the carrier mobilities show an increase by a factor of 5 as presented in Fig. 3. The highest experimentally observed conducting holes mobilities in SnGdTe correspond almost to the values known in SnTe matrix. No such increase is observed for the samples with $x>0.05$. The samples with high carrier mobility show also a considerable temperature dependence of the mobility. This is usually not observed in SnTe-based semimagnetic 
semiconductors due to a very high concentration of defects leading to the temperature independent scattering cross-section.

The experimental results presented above provide the basis for the new model of Gd ion in SnTe crystal. In this model, the $4 f^{7}$ states of $\mathrm{Gd}$ are located well below the top of the valence band, being very well localized and creating the $S=7 / 2$ magnetic moment of $\mathrm{Gd}$ ions. The key element of the model is the location of $\mathrm{Gd} 5 d^{1}$ electron states at the energy level $E_{0}\left(\mathrm{Gd}^{2+/ 3+}\right.$ level) about $0.2 \mathrm{eV}$ below the top of the valence band. It creates a new situation with Gd being in $3+$ or in $2+$ charge state depending on the position of the Fermi level with respect to the $E_{0}$ energy level. This assumption has been recently supported by the results of the photoemission experiments [31, 32]. The density of states related to $4 f^{7}$ energy states was found $9.5 \mathrm{eV}$ below the top of the valence band.

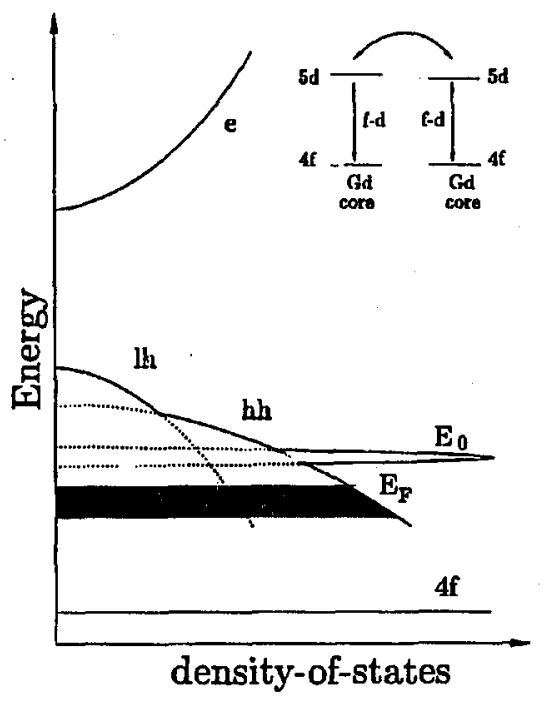

Fig. 4. The scheme of the band structure model of SnGdTe. $E_{0}$ slows the position of the $\mathrm{Gd}^{2+/ 3+}$ level and $E_{\mathrm{F}}$ indicates the position of the Fermi level, Ih is the light hole band, hh is the heavy hole band, e is the conduction band. The line labeled $4 f$ shows (not to scale) the location of the energy states of Gd $4 f$ electrons. The inset presents the scheme of the $f-d-d-f$ exchange mechanism discussed in the text.

The scheme of the band structure of SnGdTe is presented in Fig. 4. The density of states presented in this figure and related to $4 f^{7}$ electrons corresponds to the lower (spin-up) part of the total density of $4 f$ states. The spin-down half of it is located in the conduction band. The density of states assigned in this model to $5 d$ electron states (centered at the $E_{0}$ level shown in Fig. 4) represents only the lowest $t_{2 \mathrm{~g}}$ level. This orbital may be empty (corresponding to the usual case of $4 f^{7} 5 d^{0}, \mathrm{Gd}^{3+}$ electron configuration) or occupied by just one electron (the case of $\mathrm{Gd}^{2+}$ with $4 f^{7} 5 d^{1}$ configuration). 
The band structure model presented in Fig. 4 provides a basis for the consistent interpretation of both magnetic and transport properties of SnGdTe. Because of the strong localization of the $4 f$ orbitals the usual superexchange interaction via the orbitals of intervening anions is expected to be weak, as observed in other Gd- and Eu-based semimagnetic semiconductors [19]. Also the RKKY mechanism due to the $p-f$ exchange coupling between $f$ electrons and conducting holes is quite weak because the $p-f$ exchange integrals are known to be very small. The new mechanism of the coupling between the $4 f$ spins of Gd ions in SnTe (proposed in Ref. [13]) consist of two steps: the intra-ion $4 f-5 d$ exchange interaction and the inter-ion $5 d-5 d$ coupling which may be of both direct and indirect origin. The first step is known from spectroscopic data on free ions of Gd. The second step is also expected to be effective because of the considerably less localized character of $5 d$ orbitals as compared to $4 f$ and $3 d$ orbitals.

Model calculations of this two-stage mechanism with the free carriers mediating the $5 d-5 d$ coupling give the interspin exchange interaction which, in the limit of large interspin distances $(R)$ and the Fermi level below the $E_{0}$ level, is described by the formula: $I_{f f} \propto m^{*} /\left(E_{\mathrm{F}}-E_{0}\right)^{2} \cos \left(2 k_{\mathrm{F}} R\right) / R^{3}$, where $m^{*}$ is the effective mass of carriers. The theory based on the above simple model gives the correct sign of the paramagnetic Curie temperature and qualitatively describes its changes with the shift of the Fermi level [33].

The location of the $E_{0}$ level of $\mathrm{Gd}$ in the valence band of SnGdTe strongly limits the donor action of $\mathrm{Gd}$ ions in this matrix. For the Fermi level location at or above the $E_{0}\left(\mathrm{Gd}^{2+/ 3+}\right)$ level the introduction of extra $\mathrm{Gd}$ ions will result in no effect on Fermi level because they all will be in electrically neutral $2+$ charge state. The self-ionization of $\mathrm{Gd}^{2+}\left(4 f^{7} 5 d^{1}\right)$ ion to $\mathrm{Gd}^{3+}\left(4 f^{7} 5 d^{0}\right)$ would in such a case result in the increase in the energy of the system as the lowest available empty electron states are above the Fermi level. One observes experimentally only $p$-type conductivity in $\mathrm{Sn}_{1-x} \mathrm{Gd}_{x} \mathrm{Te}$ and in $\mathrm{Pb}_{1-x-y} \mathrm{Sn}_{y} \mathrm{Gd}_{x} \mathrm{Te}$ with $y>0.6$. The doping with $\mathrm{Gd}$ leads to $n$-type conductivity in $\mathrm{Pb}_{1-x} \mathrm{Gd}_{x} \mathrm{Te}$ and in $\mathrm{Pb}_{1-x-y} \mathrm{Sn}_{y} \mathrm{Gd}_{x} \mathrm{Te}$ with $y<0.6$ where the $E_{0}$ level is expected to be in the conduction band [34].

The mobility of carriers in SnGdTe is at low temperatures determined by two main scattering mechanisms, i.e., the scattering on $\mathrm{Sn}$ vacancies and on $\mathrm{Gd}$ ions. The $\mathrm{Gd}^{3+}$ ions are expected to be much more efficient scattering centers than $\mathrm{Gd}^{2+}$ ions for two reasons. First, the electrically active $\mathrm{Gd}^{3+}$ ions contribute to the scattering via their Coulomb potential absent for, neutral in SnTe lattice, $\mathrm{Gd}^{2+}$ ions. Second, the charged ion is expected to influence the local order in the matrix stronger than the neutral one. That results in a larger cross-section for the scattering by core potential. Both effects lead to the increase in the carrier mobility for samples with $E_{\mathrm{F}} \simeq E_{0}$ as it corresponds to the 2+ charge state of $\mathrm{Gd}$ ions in contrast to $3+$ charge state expected for $E_{\mathrm{F}}<E_{0}$.

The changes of the magnetic properties of $\mathrm{Sn}_{1-x} \mathrm{Gd}_{x} \mathrm{Te}$ with different $\mathrm{Gd}$ compositions are, in our model, the consequence of the Gd composition dependence of the relative position of the top of the valence band and the $E_{0}$ level of Gd. The scheme of the composition dependence of the band structure of SnGdTe which provides the basis for the explanation of the experimental results accumulated so far is presented in Fig. 5 [35]. For the Gd concentration $0.025<x<0.05$ the 


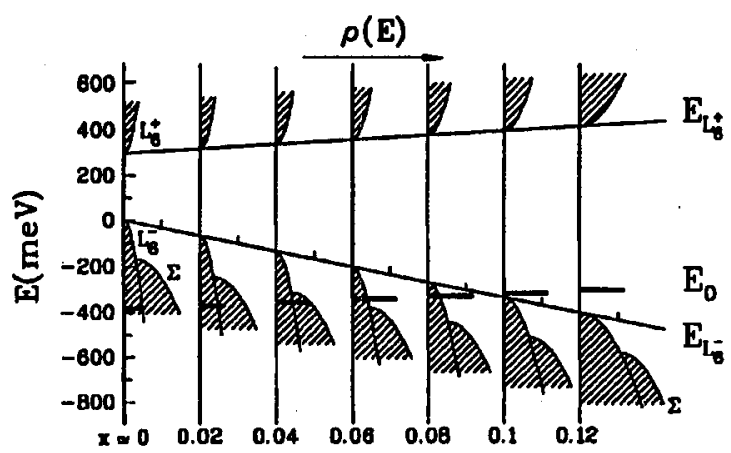

Fig. 5. The model of the composition dependence of the band structure of $\mathrm{Sn}_{1-x} \mathrm{Gd}_{x} \mathrm{Te}$. $L_{6}^{-}$and $L_{6}^{+}$label the position of the top of the valence band and the bottom of the conduction band, respectively. $\Sigma$ indicates the band of heavy holes and $E_{0}$ is the $\mathrm{Gd}^{2+/ 3+}$ energy level.

$E_{0}$ level is located in the energy range allowing for the creation of the resonance $E_{\mathrm{F}} \simeq E_{0}$ by controlling the concentration of carriers and, simultaneously, having the high density of states at the Fermi level. With increasing $\mathrm{Gd}$ content the $E_{0}$ level of Gd shifts closer to the top of the valence band and, for $x>0.05$, is located above the top of the $\Sigma$-band of heavy holes. It is now resonant with the $L$-band of light holes only and the $f-d-d-f$ exchange interaction is weak because of the low density of states at the Fermi level. With decreasing the Gd content the $E_{0}$ level moves away from the top of the valence band and, for approximately $x \leq 0.02$, is located in the energy range not accessible by Fermi level positions encountered in either as grown or annealed crystals of SnGdTe. No resonance $E_{\mathrm{F}} \simeq E_{0}$ can be created and, consequently, the magnetic properties of SnGdTe with these Gd concentrations are not expected to depend on the concentration of carriers as is indeed experimentally observed [35]. The Gd concentration induced shift of the relative position of $\mathrm{Gd} E_{0}$ level and the top of the valence band assumed in our model is quite plausible in view of the, mentioned in the Introduction, strong increase in the energy gaps of IV-VI semiconductors with the increasing concentration of rare earth ions $[4,5]$.

The above-mentioned Gd composition dependence of the position of the top of the valence band of $\mathrm{Sn}_{1-x} \mathrm{Gd}_{x} \mathrm{Te}$ with respect to the $E_{0}$ level of $\mathrm{Gd}$ has an interesting limit for $x \rightarrow 0$. One expects the $E_{0}$ level to be located below the Fermi level, i.e., the Gd ions to be always in $2+$ charge state. This prediction might be verified experimentally by electron paramagnetic resonance measurements. The preliminary results have, indeed, shown that instead of the well-known characteristic 7-line anisotropic structure of $\mathrm{Gd}^{3+}$ (observed, e.g., in PbTe:Gd [36, 37]), one observes in the SnTe:Gd single quite narrow isotropic line with $g$-factor $g=2.0$ [38].

\section{Summary}

The unique property of IV-VI semiconductors to form semimagnetic substitutional solid solutions with rare earth chalcogenides has resulted in important 
applications of the IV-VI semiconductors with Eu in the field of infrared lasers and detectors as well as provided the possibility to study the range of interesting physical effects.

We have presented, in particular, the experimental evidence and the analysis of the resonant-like Fermi level position dependence of the antiferromagnetic $f-f$ exchange interactions between Gd ions in $\mathrm{Sn}_{1-x} \mathrm{Gd}_{x} \mathrm{Te}$. The interpretation of this new effect is based on the model of the two-stage indirect $f-f$ coupling via $f-d$ intra-ion and $d-d$ inter-ion coupling via valence band electrons. Both the strong increase in the $f-f$ antiferromagnetic exchange interactions and the transport properties of SnGdTe have been consistently explained based on the new model of $\mathrm{Gd}$ ion in SnTe. The key point of the model is the location of the $\mathrm{Gd} 5 d^{1}$ electron states close to the Fermi level of SnGdTe. The main mechanism for the observed effects is the Fermi level position controlled population of $\mathrm{Gd} 5 d$ orbitals.

\section{Acknowledgments}

I would like to acknowledge the fruitful cooperation with Prof. R.R. Gałazka, M. Arciszewska, W. Dobrowolski, Z. Gołacki, M. Górska, E. Grodzicka, A. Łusakowski and B. Witkowska.

\section{References}

[1] R.R. Gałązka, J. Magn. Magn. Mater. 140-144, 13 (1995).

[2] Z. Feit, M. McDonald, R.J. Woods, V. Archambault, P. Mak, Appl. Phys. Lett. 68, 738 (1996).

[3] M. Tacke, Infrared Phys. Tech. 36, 447 (1995).

[4] G. Bauer, H. Pascher, W. Zawadzki, Semicond. Sci. Technol. 7, 703 (1992).

[5] W.C. Goltsos, A.V. Nurmikko, D.L. Partin, Solid State Commun. 59, 183 (1986).

[6] G. Karczewski, J.K. Furdyna, D.L. Partin, C.N. Thrush, J.P. Heremans, Phys. Rev. $B$ 46, 13331 (1992).

[7] G. Nimtz, B. Schlicht, R. Dornhaus, Narrow Gap Semiconductors, Springer, Berlin 1983.

[8] T. Story, G. Karczewski, L. Świerkowski, R.R. Gałązka, Phys. Rev. B 42, 10477 (1990).

[9] W.J.M. de Jonge, H.J.M. Swagten, J. Magn. Magn. Mater. 100, 322 (1991).

[10] W.J.M. de Jonge, T. Story, H.J.M. Swagten, P.J.T. Eggenkamp, Europhys. Lett. 17, 631 (1992).

[11] H.J.M. Swagten, W.J.M. de Jonge, R.R. Gałązka, P. Warmenbol, J.T. Devreese, Phys. Rev. B 37, 9907 (1988).

[12] T. Story, C.H.W. Swüste, P.J.T. Eggenkamp, H.J.M. Swagten, W.J.M. de Jonge, Phys. Rev. Lett. 77, 2802 (1996).

[13] T. Story, M. Górska, A. Lusakowski, M. Arciszewska, W. Dobrowolski, E. Grodzicka, Z. Golacki, R.R. Gałązka, Phys. Rev. Lett. 77, 3447 (1996).

[14] M. Górska, T. Story, M. Arciszewska, E. Grodzicka, Z. Gołacki, A. Eusakowski, Acta Phys. Pol. A 87, 197 (1995).

[15] T. Story, M. Górska, M. Arciszewska, Z. Gołacki, R.R. Gałązka, J. Magn. Magn. Mater. 140-144, 2041 (1995). 
[16] W. Dobrowolski, E. Grodzicka, T. Story, Z. Gołacki, R.R. Gałązka, in: Narrow Gap Semiconductors 1995, Ed. J.L. Reno, IOP Publishing, Bristol 1995, p. 125.

[17] P. Urban, G. Sperlich, Solid State Commun. 16, 927 (1975).

[18] T. Dietl, C. Śliwa, G. Bauer, H. Pascher, Phys. Rev. B 49, 2230 (1994).

[19] M. Górska, J.R. Anderson, G. Kido, S.M. Green, Z. Gołacki, Phys. Rev. B 45, 11702 (1992).

[20] V. Bindilatti, N.F. Oliveira, Y. Shapira, G.H. McCabe, M.T. Liu, S. Isaber, S. Charar, M. Averous, E.J. McNiff, Z. Golacki, Phys. Rev. B 53, 5472 (1996).

[21] M. Górska, J.R. Anderson, J.L. Peng, Y. Oka, J.Y.-Jen, I. Mogi, D. Ravot, Z. Gołacki, Phys. Rev. B 55, 4400 (1997).

[22] S. Isber, S. Charar, X. Gratens, C. Fau, M. Averous, S. Misra, Z. Gołacki, Phys. Rev. B 54, 7634 (1996).

[23] T. Story, C.H.W. Swüste, H.J.M. Swagten, W.J.M. de Jonge, to be published.

[24] R. Denecke, L. Ley, G. Springholz, G. Bauer, Phys. Rev. B 53, 4534 (1996).

[25] Y. Iida, T. Shimizu, H. Enomoto, II. Ozaki, Jpn. J. Appl. Phys. 32, 4449 (1993).

[26] D.L. Partin, J. Vac. Sci. Technol. B 1, 174 (1983).

[27] S.K. Das, R. Suryanarayanen, J. Appl. Phys. 66, 484 (1989).

[28] F. Mahoukou, O. Dos Santos, C. Fau, S. Charar, M. Averous, Z. Gołacki, Phys. Status Solidi B 195, 511 (1996).

[29] S. Isaber, S. Charar, C. Fau, V. Mathet, M. Averous, Z. Golacki, Phys. Rev. B 52, 1678 (1995).

[30] E. Grodzicka, W. Dobrowolski, T. Story, E.I. Slynko, Yu.K. Vygranenko, M.M.H. Willekens, H.J.M. Swagten, W.J.M. de Jonge, Acta Phys. Pol. A 90, 801 (1996).

[31] B.A. Orłowski, B.J. Kowalski, Z. Gołacki, T. Story, R.L. Johnson, Acta Phys. Pol. $A$ 88, 857 (1995).

[32] B.J. Kowalski, Z. Gołacki, E. Guziewicz, B.A. Orłowski, R.L. Johnson, Acta Phys. Pol. A 92, (1997), Proc. of this Conf. (Part II).

[33] A. Łusakowski, Acta Phys. Pol. A 88, 1018 (1995).

[34] T. Story, M. Arciszewska, P. Eazarczyk, A. Łusakowski, M. Górska, W. Dobrowolski, B. Witkowska, E. Grodzicka, R.R. Gałazka, Acta Phys. Pol. A 92, (1997), Proc. of this Conf. (Part II).

[35] T. Story, M. Arciszewska, W. Dobrowolski, Z. Gołacki, M. Górska, E. Grodzicka, A. Łusakowski, E. Dynowska, B. Witkowska, Acta Phys. Pol. A 90, 935 (1996).

[36] M. Bartkowski, D.J. Northcott, A.H. Reddoch, Phys. Rev. B 34, 6506 (1986).

[37] A. Bruno, J.P. Lascaray, M. Averous, G. Fillion, J.F. Dumas, Phys. Rev. B 37, 1186 (1988).

[38] T. Story, A. Eusakowski, C.H.W. Swüste, H.J.M. Swagten, W.J.M. de Jonge, to be published. 